\title{
POLYPROPYLENE FILMS STUDIED WITH VARIABLE POSITRON BEAMS
}

\author{
M. Degbowska ${ }^{a}$, P.G. Coleman ${ }^{b}$ and D.W. Gidley ${ }^{c}$ \\ ${ }^{a}$ Institute of Experimental Physics, University of Wrocław \\ Pl. Maksa Borna 9, 50-204 Wrocław, Poland \\ ${ }^{b}$ School of Physics, University of East Anglia, Norwich NR4 7TJ, United Kingdom \\ ${ }^{c}$ Department of Physics, University of Michigan, Ann Arbor, MI 48109, USA
}

Doppler broadening and positron lifetime spectra have been measured for polypropylene films of constant thickness $(\approx 50 \mu \mathrm{m})$ and different morphology that crystallized from melt at temperature 0,100 , and $120^{\circ} \mathrm{C}$. The $S$-parameter vs. energy curves do not change in a systematic way with crystallinity of samples. It is supposed that decrease in the fractional free volume for samples of greater crystallinity is not seen in $S$-values because of their increase resulting from annihilation in defects present both in the crystalline regions and on the lamellae surfaces. Intensities of the components in the positron lifetime spectra: $I_{2}\left(\tau_{2}=1.3 \mathrm{~ns}\right)$ and $I_{3}\left(\tau_{3} \approx 2.3 \div 2.8 \mathrm{~ns}\right)$ show changes with crystallinity of samples. The evident trend is observed for the longer-lived $\tau_{3}$-component intensity to decrease with crystallinity while the reverse is true for the shorter, fixed $\tau_{2}$-component. The decrease in the intensity of the longer-lived component can result from reduction of the fractional free volume.

PACS numbers: $36.10 . \mathrm{Dr}, 61.41 .+\mathrm{e}, 71.60 .+\mathrm{z}, 78.70 . \mathrm{Bj}$

\section{Introduction}

Polypropylene, a member of the first class of vinyl polymers, is semicrystalline [1]. When the isotactic form is cooled from the molten state, "the spherulitic crystallization" takes place giving rise to spherulites visible under a polarizing microscope. They are aggregates of plate-like crystallites (lamellae) with folded chains pervaded with amorphous material (interlamellar amorphous phase) [2]. The amorphous phase is present also between spherulites (interspherulitic amorphous phase). When controlling the kinetics of the crystallization one can produce samples not only of different crystallinity but also of different morphology (i.e. of different mean size of spherulites, perfection and amount of interspherulitic and interlamellar amorphous phase fractions). The degree of crystallinity determines chemical, thermo-mechanical, and electrical properties of the polymer. Its density increases with crystallinity [3]. In Refs. [4, 5] the thermooxidative degradation of isotactic polypropylene thin films of different degree of crystallinity and varying morphology was studied in air. It was found that both the rates of degradation and the following structural changes depend not only on the initial crystallinity 
but also on morphology of the samples under study. A model was given [5] in which the thermooxidation was considered as a complex reaction occurring in the interspherulitic amorphous regions (more easily accessible to the oxygen) as well as in the interlamellar amorphous regions (the slower oxidation rate, controlled by diffusion of oxygen into the spherulite). The fractional free volume, $f$, in the samples was assumed to change with crystallinity $X_{\mathrm{c}}$ according to the formula

$$
f=f_{0}\left(1-X_{\mathbf{c}}\right)^{0.5}
$$

where $f_{0}$ denotes the fractional free volume in the amorphous polypropylene.

In the case of bulk semicrystalline polymers two long-lived components often occur in positron annihilation lifetime spectra; one with the lifetime close to $1 \mathrm{~ns}$ and the longest-lived one with the lifetime of a few nanoseconds (cf. $[6 \div 18]$ and references therein). It is generally accepted that the longest lifetime corresponds to the average pick-off lifetime of $o$-Ps in cavities in an amorphous phase while the origin and physical meaning of the shorter one is still under debate [13]. Studies of bulk polypropylene performed at room temperature [16] show two distinct long lifetimes of about 1.3 and $2.7 \mathrm{~ns}$, each with roughly $10 \%$ intensity.

In Refs. $[17,18]$ both the intermediate and the longest-lived component were assumed to originate from o-positronium annihilation by pick-off in ordered and . amorphous phases, respectively, and positron annihilation lifetime spectra were well fitted using a simple model involving positronium diffusion and trapping from the crystalline to amorphous regions in lamellar polyethylene samples. In Ref. [19] results of positron annihilation lifetime measurements for semicrystalline (lamellar) samples (with spherulites) of poly(aryl-ether-ether ketone) were given but only one long-lived component was revealed with the lifetime of $\approx 1.8 \mathrm{~ns}$ and intensity decreasing with rise of crystallinity of the studied samples.

The aim of this paper was to check if varying crystallinity and morphology of thin films of isotactic polypropylene influences positron annihilation characteristics determined with use of monoenergetic positron beams. Such beams have already been used to study polymer foils [20-33].

\section{Samples}

\subsection{Crystallization from melt}

Three foils of isotactic polypropylene (PP Novolen $1100 \mathrm{H}$ ), that were melted during 5 min at $185^{\circ} \mathrm{C}$ between two metal plates under pressure of $3 \times 10^{8} \mathrm{~N} / \mathrm{m}^{2}$, crystallized under isothermal conditions in $5 \mathrm{~min}$ at temperature 0,100 , and $120^{\circ} \mathrm{C}$. Three different baths were used: melting ice, distilled water, and silicon oil, respectively. The samples will be denoted further in the text as PP0, PP100, and PP120.

\subsection{Crystallinity of samples}

Crystallinity of samples was studied with the use of wide angle X-ray scattering (WAXS). The WAXS measurements were carried out with a HZG diffractometer using $\mathrm{Cu} K_{\alpha}$ filtered radiation (wavelength $=0.154 \mathrm{~nm}$ ) in the diffraction angle range $2 \Theta=5 \div 40^{\circ}$. The method developed by Hindeleh and Johnson [34] was used to extract contributions of two components (due to scattering from crystalline and amorphous regions; i.e. peaks and the so-called "amorphous halo"). 
The fractional çrystallinity, giving in that case information on the mass contribution of the crystalline phase, $X_{\mathrm{m}}$, was calculated as the ratio of the area under the resolved peaks to the total area under the experimental diffraction pattern. One crystal structure only was identified in the samples PP0 and PP100 ( $\alpha$ form; monoclinic), while in the case of PP120 the $\beta$ form (hexagonal) was recognized, too. The data from the WAXS measurements are given in Table I.

\section{TABLE I}

Results of WAXS measurements.

\begin{tabular}{l|c|c|c}
\hline \hline Sample & \multicolumn{2}{|c|}{ Content } & \multirow{2}{*}{$X_{\mathrm{m}}$} \\
\cline { 2 - 3 } & $\alpha$ phase & $\beta$ phase & \\
\hline PP0 & 0.410 & 0 & 0.410 \\
PP100 & 0.504 & 0 & 0.504 \\
PP120 & 0.460 & 0.072 & 0.532
\end{tabular}

\subsection{IR absorption measurements}

Infrared spectra were obtained with a Nicolet "Magna 860" Fourier transform spectrometer using both the transmission and attenuation of total reflection (ATR) technique. The equipment was set to operate in the range $400-5000 \mathrm{~cm}^{-1}$ with a resolution of $4 \mathrm{~cm}^{-1}$, obtaining a spectrum that represented the average of 100 scans. The depth of penetration of the samples by the reflected IR radiation (in case of the ATR technique) was between 8 and $13 \mu \mathrm{m}$. Changes were observed between 1250 and $800 \mathrm{~cm}^{-1}$ that suggest the formation of the epi-oxide structures while the broad band in the region of $1100 \div 1000 \mathrm{~cm}^{-1}$, the ether-like structures. The highest increase in the region occurred for the sample PP120. Most probably the oxygen bridges crosslinking the polimer are revealed there. In addition, an increase near $1650 \mathrm{~cm}^{-1}$ was observed reflecting the formation of small number of double bonds (between carbon atoms). The results prove the possibility of oxidation of the subsurface regions of samples especially effective in the case of the sample PP120.

\subsection{Spherulites by polarizing microscope}

The samples were observed in halogenic, polarized, transmitted and reflected light by using an optical polarizing microscope OPTIPHOT 2-POL from Nikon Corporation. A monochromatic blue filter and planapochromatic objectives DIC with the highest degree of correlation of all aberrations were used.

Because of optical side-effects, the samples of polymers were studied without any cover glass and kollolith. The studies were realized with 50, 200, 400, and 1000 magnification. Microscopic pictures were recorded in PC-computer. Size analysis of spherulites was performed using the computer program "Lucia-M" from Laboratory Imaging Ltd. (Czech Republic). In the sample PP120 the largest spherulites exist. The observations allowed to estimate the average value of the longest diagonal of the spherulites as equal to 6,12 , and $50 \mu \mathrm{m}$ in PP0, PP100, and PP120. 
The basic substructure of crystallites is in form of lamellae of folded chains. Their characterization was done by the small angle X-ray scattering (SAXS) technique.

\subsection{Morphology of samples studies by SAXS technique}

The SAXS investigations were performed within the range $0 \div 5^{\circ}$ by means of a MBraun (Austria) SWAX camera which utilizes the conventional Kratky collimation system. $\mathrm{Cu} K_{\alpha}$ radiation was used; monochromatization was performed by a Ni $\beta$ filter and a pulse-height discrimination. The experimental SAXS curves were corrected for sample absorption and desmeared from collimation distortion by means of the computer program 3D VIEW supplied by MBraun. Analysis of the SAXS curves was based on the standard theory for pseudo-two-phase lamellar systems [35]. It has been assumed that the samples under investigation contain alternating crystalline and amorphous regions placed in stacks. Both phases are connected by a transition layer in which the electron density varies linearly from $\rho_{\mathrm{c}}$ (the density of the crystalline phase) to $\rho_{\mathrm{a}}$ (the density of the amorphous phase). The one-dimensional correlation function (the Fourier transform of the SAXS curve) gave information on the long period $L$, the number average value of the thickness of the crystalline layers $l_{c}$ and the thickness $E$ of the transition layer between the crystalline and amorphous regions. The values of $L, l_{c}$, and $E$ are given in Table II together with the volume contribution of the crystalline regions; $X_{\mathrm{v}}$, determined from the SAXS measurements.

\section{TABLE II}

Results of the SAXS measurements.

\begin{tabular}{l|c|c|c|c}
\hline \hline Sample & $L[\AA]$ & $l_{\mathrm{c}}[\AA]$ & $E[\AA]$ & $X_{\mathrm{v}}$ \\
\hline PP0 & 104 & 33 & 9.3 & 0.133 \\
PP100 & 140 & 46 & 8.4 & 0.175 \\
PP120 & 200 & 58 & 9.8 & 0.180
\end{tabular}

Further details of the analysis of the SAXS data will be given elsewhere [36]. Large differences are seen between the values of $X_{\mathrm{m}}$ and $X_{\mathrm{v}}$ but they do not exceed the maximum value of the difference allowable by the Sheldon equation [37].

\section{Measurements of Doppler broadening of annihilation line and positron lifetime spectra}

\subsection{Doppler broadening}

The variable-energy Doppler broadening measurements were performed for all specimens at room temperature using a computer-controlled magnetic-transport beam system at UEA Norwich [38]. (A comprehensive review of positron beam techniques and their application is presented in [39].) Incident positron energies $E$ varied between 0.1 and $29 \mathrm{keV}$. Energy spectra of annihilation gamma rays were measured with a Ge detector having an energy resolution (FWHM) of about $1.2 \mathrm{keV}$ at $511 \mathrm{keV}$. 
The polypropylene samples were held on a stainless steel holder. A current was passed through a nearby tungsten filament during experimental runs, in order to bathe the sample surface with electrons to counteract charging effects seen in earlier measurements on insulating films [27]. Approximately $10^{6}$ counts were accumulated in a photopeak at each incident positron energy $E$ in time of $500 \mathrm{~s}$.

The Doppler broadening of the annihilation line (caused by the momentum of the annihilating electron-positron pair) is characterized by the line-shape parameter $S$. The value of $S$ is defined as the integral of gamma ray intensity in the central energy region divided by the total intensity of the line. A rise in $S$ implies a narrowing of the line. Such narrowing occurs when for instance vacancies [40], large open volume defects (voids) $[41,42]$ or pores are present in a sample [43].

\subsection{Positron lifetime spectra}

Positron lifetime spectra were acquired using a variable energy, UHV positron beam as described in [22]. The beam is directed at a channel plate detector that is tilted at 45 degrees with respect to the beam where a retarding electric field deflects the positrons into the sample. Secondary electrons from the sample are detected in the channel plate as a "start" signal. The gamma "stop" detector and associated electronics are similar to conventional fast timing systems. The time resolution of this system has been improved to $0.35 \mathrm{~ns}$. Two beam implantation energies of $2.2 \mathrm{keV}$ and $4 \mathrm{keV}$ were used with the three polypropylene films. The thickness of the films caused some beam-related charge-up problems to degrade the time resolution somewhat, but this problem was minimized by heating a nearby filament once every two hours during a typical 6 hour run. The total number of events recorded in each spectrum is $3-4$ millions. The $2.2 \mathrm{keV}$ spectra were acquired using a TAC-ADC timing system with $38 \mathrm{ps} / \mathrm{channel}$. As a systematic check, the $4 \mathrm{keV}$ data were acquired using a digital timer with 16000 channels of 156 ps width.

\section{Results of positron annihilation measurements. Discussion}

The $S$ parameter vs. incident positron energy is shown for all studied samples in Fig. 1. The variation of the temperature of crystallization of films does not result in systematic changes of $S$-values characteristic of them. One could expect from (1) greater values of $S$ for a sample that crystallized at lower temperature and having lower degree of crystallinity while the sequence of our $S-E$ curves for samples PP100 and PP120 do not follow the supposition. For each studied sample a tendency of lowering of the $S$-values is seen for higher energies of the incident positrons. In the case of the sample PP120 the lowering of $S$-value begins at the pronounced threshold energy $\approx 10 \mathrm{keV}$ that corresponds to the average positron implantation depth of $\approx 2 \mu \mathrm{m}$ in the sample.

The program VEPFIT [44] was used to fit the experimental results. The samples were modelled as consisting of two layers of different $S$-values that were fitted together with positron diffusion length in the layers and the layer boundaries. Only in the case of the sample PP120 the variance of the fit was satisfactory: The thickness of the upper layer was fitted as equal to $(1.7 \pm 2.3) \mu \mathrm{m}$. Other parameters (except $S$-values) were fitted with high errors. The fitted values of the thickness 


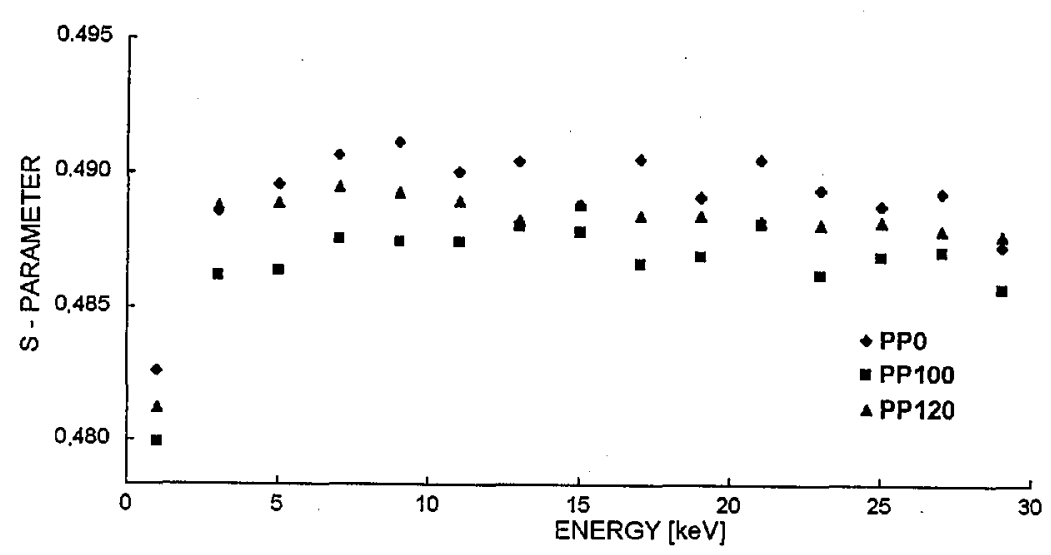

Fig. 1. $S$-parameter vs. incident positrons energy.

of the upper layer in the case of samples PP0 and PP100 exceeded $10 \mu \mathrm{m}$ (a little smaller value 'was obtained for PP100). The idea of differentiation of the most outside parts of the film from its center, originated from the observed decrease in $S$-values at higher values of the energy of the implanted positrons as well as from the results of ATR measurements, suggesting the possibility of the oxidation of the subsurface region of the samples. It is worthy taking into account a model of crystallization from the melt mentioned in [1], p. 603. It is predicted that when a homogeneous polymer melt (placed for instance between two metal plates as in our case) is suddenly brought in contact with a cooler bath, the spatial size distribution of spherulites occurs ("the larger the distance from the metal, the lower the speed of cooling, the larger size of the spherulitic grains"). Therefore one may expect that in the case of our samples the sub-surface region of them is quite different from the center of the samples as far as the degree of crystallinity and the fractional content of the amorphous phase (both the intra- and interspherulitic) is considered. The question is how to study such changes. What is more, defects known to occur in crystalline regions and both at lammellae and the spherulite surface, give contributions to the $S$-values that may result in their increase despite the reduction of the fractional free volume following the rise in crystallinity.

The best fitting (lowest chi-square) of the lifetime spectra was achieved using 4 lifetime components with absolutely no constraints. The fitted lifetimes (intensities) are about $0.38 \mathrm{~ns}(75 \%), 1.8 \mathrm{~ns}(18 \%), 3.7 \mathrm{~ns}(6 \%)$, and $80 \mathrm{~ns}(1 \%)$. The shortest lifetime component is almost certainly a convolution of two short components, one near $125 \mathrm{ps}$ and another close to 400 ps that account for parapositronium and positrons annihilating in the films. The long component is unique to beam-PALS studies and is due to backscattered positrons forming positronium in the vacuum region between the sample and the channel plate (see [22]). Unfortunately, it is known that such backscattering also produces an intermediate lifetime component that is crudely approximated by a 7-8 ns exponential. Thus the fitted $3.7 \mathrm{~ns}$ component with only $6 \%$ intensity is certainly affected by such events. Such uncon- 
strained, 4-component fitting produced the lowest chi-square values and showed no trend with film crystallinity. However, bulk studies of polypropylene at room temperature [16] show two distinct long lifetimes of about $1.3 \mathrm{~ns}$ and $2.7 \mathrm{~ns}$, each with roughly $10 \%$ intensity. An unconstrained fitting with 5 lifetime components did not converge on meaningful values, however we did get consistent results when we constrained the longest lifetimes to $80 \mathrm{~ns}$ and $7.5 \mathrm{~ns}$, and fixed a lifetime at $1.3 \mathrm{~ns}\left(\tau_{2}\right)$. The fitted intermediate lifetime $\left(\tau_{3}\right)$ is then found to be $2.4-2.8 \mathrm{~ns}$ in good agreement with bulk results. The detailed results at both energies are given in Table III.

\section{TABLE III}

Intermediate components in the lifetime spectra.

\begin{tabular}{l|c|c|c|c}
\hline \hline Sample & $\tau_{2}[\mathrm{~ns}]$ & $I_{2}[\%]$ & $\tau_{3}[\mathrm{~ns}]$ & $I_{3}[\%]$ \\
\hline \multicolumn{5}{c}{ Energy $2.2 \mathrm{keV}$} \\
\hline PP0 & 1.3 & $8.7 \pm 0.8$ & $2.45 \pm 0.03$ & $13.8 \pm 0.5$ \\
PP100 & 1.3 & $9.0 \pm 0.7$ & $2.50 \pm 0.03$ & $14.1 \pm 0.5$ \\
PP120 & 1.3 & $11.5 \pm 0.7$ & $2.80 \pm 0.04$ & $11.7 \pm 0.5$ \\
\hline \multicolumn{5}{c}{ Energy 4 keV } \\
\hline PP0 & 1.3 & $4.6 \pm 0.7$ & $2.27 \pm 0.02$ & $18.5 \pm 0.5$ \\
PP100 & 1.3 & $7.8 \pm 0.6$ & $2.42 \pm 0.02$ & $16.7 \pm 0.4$ \\
PP120 & 1.3 & $9.2 \pm 0.5$ & $2.59 \pm 0.02$ & $15.4 \pm 0.3$
\end{tabular}

The evident trend is for the longer component intensity $\left(I_{3}\right)$ to decrease with crystallinity while the reverse is true for the shorter, fixed lifetime component $\left(I_{2}\right)$. Bulk studies claim [16] that the longer component is related to positronium decay in the amorphous regions and our observed trend is consistent with that interpretation. Bulk studies have not pinned down the interpretation of the $1.3 \mathrm{~ns}$ component, but our results suggest that it is related to positronium decay in the crystalline polymer regions. More quantitatively, we find that the long component intensity decreases by $17 \pm 3 \%$ and $15 \pm 5$ for the $4 \mathrm{keV}$ and $2.2 \mathrm{keV}$ data respectively when the contribution of the amorphous phase decreases by $20 \%$. The $1.3 \mathrm{~ns}$ component intensity is much more variable, but trends upwards with crystallinity.

One can see also the changes of the positron lifetime parameters given in Table III with the energy of the implanted positrons. The more deeply they penetrate the film, the less is the value of $\tau_{3}$ and $I_{2}$, while $I_{3}$ increases for each sample. The variations may be a reflection of changes in morphology of samples along the distance from the surface mentioned above. Though these lifetime studies only probed the top $1 \mu \mathrm{m}$ of the sample, it would be interesting in future beam-PALS work to use much higher $(30 \mathrm{keV})$ beam implantation energies. Changes in $\tau_{3}$ and $I_{3}$ with the energy of positrons have already been observed (in [29] and [22] correspondingly) while the $\tau_{2}$-component and the changes of $I_{2}$ and $I_{3}$ with crystallinity are to our knowledge reported for the first time in the case of studies of polymers with the slow positron beam. 


\section{Acknowledgments}

This work was partially supported by the grant 2016/W/IFD/98 of the Committee for Scientific Research (Poland). The studies at University of Michigan were supported by the National Science Foundation (USA) grant ECS-9732804. One of us (M.D.) would like to record warm thanks to the Authorities of the School of Physics of UEA for very kind hospitality during the stay at Norwich. The technical assistance of Farhad Malik, a PhD student in School of Physics of UEA is greatly appreciated. Professor Maria Mucha from the Technical University in Łódź (Poland) is greatly acknowledged for giving the idea of studies of polypropylene films, discussions, and cooperation.

\section{References}

[1] D.W. van Krevelen, Properties of Polymers, Elsevier, Amsterdam 1990, p. 558.

[2] In Ref. [1], p. 587.

[3] K. Kelar, D. Ciesielska, Fizykochemia polimerów, Politechnika Poznańska, Poz-. nań 1997 (in Polish).

[4] M. Mucha, M. Kryszewski, Colloid Polym. Sci. 258, 743 (1980).

[5] M. Mucha, Colloid Polym. Sci. 264, 1 (1986).

[6] S.J. Tao, J.H. Green, Proc. Phys. Soc. 85, 463 (1964).

[7] P. Kindl, W. Puff, H. Sormann, Phys. Status Solidi A 58, 489 (1980).

[8] P. Kindl, H. Sormann, W. Puff, in: Positron Annihilation, Eds. P.G. Coleman, S.C. Sharma, L.M. Diana, North-Holland, Amsterdam 1982, p. 685.

[9] P. Kindl, Phys. Status Solidi A 81, 293 (1984).

[10] P. Kindl, H. Sormann, W. Puff, in: Positron Annihilation, Ed. P.C. Jain, R.M. Singru, K.P. Gopinathan, World Scientific, Singapore 1985, p. 793.

[11] P. Kindl, G. Reiter, Phys. Status Solidi A 104, 707 (1987).

[12] P. Kindl, G. Reiter, in: Positron Annihilation, Ed. L. Dorikens-Vanpraet, M. Dorikens, D. Segers, World Scientific, Singapore 1989, p. 806.

[13] G. Consolati, F. Quasso, Appl. Phys. A 50, 43 (1990); O.E. Mogensen, in: Positron Annihilation in Chemistry, Springer-Verlag, Berlin 1995, p. 226 and next pages.

[14] T. Suzuki, Y. Oki, M. Numajiri, T. Miura, K. Kondo, Y. Ito, J. Polym. Sci. B 30, 517 (1992).

[15] A. Uedono, Y. Okho, S. Watauchi, Y. Ujihira, in: Proc. Int. Symp. on Material Chemistry in Nuclear Environment, Tsukuba (Japan) 1992, p. 479.

[16] M. Tanaka, K. Takebe, A. Uedono, Y. Ujihira, K. Horie, T. Asanuma, Mater. Sci. Forum 105-110, 1737 (1992); Y. Okho, A. Uedono, Y. Ujihira, J. Polym. Sci. B 33, 1183 (1995).

[17] J. Serna, J.Ch. Abbé, G. Duplâtre, Phys. Status Solidi A 115, 389 (1989).

[18] J. Serna, Phys. Status Solidi A 122, 489 (1990).

[19] H. Nakanishi, Y.C. Jean, E.G. Smith, T.C. Sandreczki, J. Polym. Sci. B 27, 1419 (1989).

[20] J.J. Singh, Nucl. Instrum. Methods Phys. Res. B 79, 349 (1993).

[21] Y. Kobayashi, I. Kojima, S. Hishita, T. Suzuki, E. Asari, M. Kitajima, Phys. Rev. B 52, 823 (1995). 
[22] L. Xie, G.B. DeMaggio, W.E. Frieze, J. DeVries, D.W. Gidley, H.A. Hristov, A.F. Yee, Phys. Rev. Lett. 74, 4947 (1995) and references therein.

[23] J. Xu, L.D. Hulett, Jr., T.A. Lewis, N.H. Tolk, Appl. Surf. Sci. 85, 49 (1995).

[24] L.D. Hulett, Jr., S. Wallace, J. Xu, B. Nielsen, Cs. Szeles, K.G. Lynn, J. Pfau, A. Schaub, Appl. Surf. Sci. 85, 334 (1995).

[25] G.B. De Maggio, W.E. Frieze, D.W. Gidley, M. Zhu, H.A. Hristov, A.F. Yee, Phys. Rev. Lett. 78, 1524 (1997).

[26] D.W. Gidley, G.B. DeMaggio, W.E. Frieze, M. Zhu, H.A. Hristov, A.F. Yee, Mater. Sci. Forum 255-257, 635 (1997).

[27] P.G. Coleman, S. Kuna, R. Grynszpan, Mater. Sci. Forum 255-257, 668 (1997).

[28] R. Zhang, H. Cao, J.P. Yuan, C.M. Huang, Q. Zhang, T.C. Sandreczki, B. Nielsen, P. Asoka-Kumar, R. Suzuki, T. Ohdaira, Y.C. Jean, Mater. Sci. Forum 255-257, 704 (1997),

[29] Y.C. Jean, H. Cao, G.H. Dai, R. Suzuki, T. Ohdaira, Y. Kobayashi, K. Hirata, Appl. Surf. Sci. 116, 251 (1997).

[31] K. Hirata, Y. Kobayashi, S. Hishita, Y. Ujihira, Appl. Phys. A 64, 491 (1997).

[32] Y.C. Yean, R.W. Zhang, H. Cao, J.P. Yuan, C.M. Huang, B. Nielsen, P. Asoka-Kumar, Phys. Rev. B 56, R8459 (1997).

[33] G. Wiśniewski, G.P. Karwasz, R.S. Brusa, A. Zecca, Nukleonika 42, 253 (1997).

[34] A.M. Hindeleh, D.J. Johnson, J. Phys. D, Appl. Phys. 4, 259 (1971).

[35] A. Włochowicz, Cz. Ślusarczyk, A. Narębska, G. Szymański, J. Macromol. Sci.-Phys. B 31, 239 (1992).

[36] M. Dębowska, Cz. Ślusarczyk, to be published.

[37] G.W. Urbańczyk, Mikrostruktura włókna, WNT, Warszawa 1988, p. 23 (in Polish).

[38] N.B. Chilton, P.G. Coleman, Meas. Sci. Technol. 6, 53 (1995).

[39] P.J. Schultz, K.G. Lynn, Rev. Mod. Phys. 60, 701 (1988).

[40] C. Dauwe, M. Dorikens, L. Dorikens-Vanpraet, D. Segers, Appl. Phys. 5, 117 (1974).

[41] A. Dupasquier, G. Ottaviani, in: Proc. Int. School of Physics "Enrico Fermi" Course CXXV, Eds. A. Dupasquier, A.P. Mills Jr., IOS Press, Amsterdam 1995, p. 614 .

[42] P.J. Mijnarends, Europhys. News 25, 178 (1994).

[43] A.P. Knights, G. Kowalski, A.S. Saleh, A. Towner, M.I. Patel, P.C. Rice-Evans, M. Moore, G.A. Gledhill, A. Nosarzewski-Orłowska, A. Brzozowski, J. Appl. Phys. 78, 4411 (1995).

[44] A. Van Veen, H. Schut, J. de Vries, R.A. Hakvoort, M.R. Ijpma, in: Positron Beams for Solids and Surfaces, Eds. P.J. Schultz, G.R. Massoumi, P.J. Simpson, American Institute of Physics, New York 1990, p. 171. 